\title{
NUMERICAL SIMULATION OF MAGNETO-HYDRODYNAMICS MIXED CONVECTION IN AN OPEN CHANNEL HAVING A SEMI- CIRCULAR HEATER
}

\author{
M. M. Billah ${ }^{1}$, M. J. H. Munshi ${ }^{2}$, A. K. Azad ${ }^{3}$, M. S. Uddin ${ }^{4}$, M. M. Rahman ${ }^{5}$ \\ ${ }^{1}$ Department of Arts and Sciences, Ahsanullah University of Science and Technology (AUST), Dhaka-1208, \\ Bangladesh, Email: mmb.edu@gmail.com/mmb.as@aust.edu \\ ${ }^{2}$ Department of Mathematics, Hamdard University Bangladesh, Gozaria, Munshiganj, Bangladesh \\ ${ }^{3}$ Department of Business Administration, International Islamic University Chittagong, DC, Bangladesh \\ ${ }^{4}$ Department of Mathematics, Jahangirnagar University, Savar, Dhaka-1342, Bangladesh \\ ${ }^{5}$ Department of Mathematics, Bangladesh University of Engineering and Technology (BUET), Dhaka-1000, \\ Bangladesh, Email: mmustafizurrahman@math.buet.ac.bd
}

\begin{abstract}
:
The present study is conducted to investigate heat and fluid flow in an open channel having a circular heater on the bottom surface under magnetic field. The bottom surface is heated isothermally, but the other walls of the channel are kept adiabatic. The consequent mathematical model is governed by the coupled equations of mass, momentum and energy and solved by employing Galerkin weighted residual method of finite-element technique. A wide range of pertinent parameters such as Rayleigh numbers $\left(10^{3} \leq \mathrm{Ra} \leq 10^{5}\right)$ and Hartmann numbers $(10 \leq H a \leq 100)$ are considered in the present study. In addition, the mixed convection regime is occurred due to buoyancy and shear forces. Various characteristics such as streamlines, isotherms and heat transfer rate in terms of the average Nusselt number $\left(N u_{a v}\right)$, average fluid temperature $\left(\theta_{a v}\right)$, and Drag force $(D)$ is investigated for the aforesaid parameters. The magnetic field is found as a control parameter on heat and fluid flow, particularly at higher Rayleigh numbers. It is observed that Hartmann numbers have a significant effect on average Nusselt number, average fluid temperature and Drag force.
\end{abstract}

Keywords: Penalty finite element method, channel flow, Magnetohydrodynamics, circular heater, open cavity.

\section{NOMENCLATURE}

$B_{0} \quad$ Magnetic induction (Tesla)

$C_{p} \quad$ Specific heat of fluid at constant pressure

$g \quad$ Gravitational acceleration $\left(\mathrm{ms}^{-2}\right)$

$\mathrm{Ha}$ Hartmann number

$\kappa \quad$ Thermal conductivity of fluid $\left(\mathrm{Wm}^{-1} \mathrm{~K}^{-1}\right)$

$L \quad$ Length of the cavity (m)

$N \quad$ The non-dimensional distances either along

$X$ or $Y$ direction acting normal to the surface

$\mathrm{Nu} \quad$ Nusselt number

$p \quad$ Dimensional pressure $\left(\mathrm{Nm}^{-2}\right)$

$P \quad$ Dimensionless pressure

Pr Prandtl number

$R a$ Rayleigh number

Re Reynolds number

$T \quad$ Dimensional temperature (K)

$u, v$ Dimensional velocity components $\left(\mathrm{ms}^{-1}\right)$

$U, V$ Dimensionless velocity components

W Height of the cavity (m)
$\bar{V} \quad$ Cavity volume $\left(\mathrm{m}^{3}\right)$

$x, y \quad$ Cartesian co-ordinates (m)

$X, Y$ Dimensionless Cartesian coordinates

Greek symbols

$\alpha \quad$ Thermal diffusivity $\left(\mathrm{m}^{2} \mathrm{~s}^{-1}\right)$

$\beta \quad$ Thermal expansion coefficient $\left(\mathrm{K}^{-1}\right)$

$v \quad$ Kinematic viscosity $\left(\mathrm{m}^{2} \mathrm{~s}^{-1}\right)$

$\theta \quad$ Non dimensional temperature

$\rho \quad$ Density of the fluid $\left(\mathrm{kgm}^{-3}\right)$

$\mu \quad$ Dynamic viscosity of the fluid $\left(\mathrm{m}^{2} \mathrm{~s}^{-1}\right)$

$\sigma \quad$ Electrical conductivity $\left(\mathrm{Sm}^{-1}\right)$

$\psi \quad$ Stream function

\section{Subscripts}

av average 
i inlet state

$h \quad$ heated wall
Abbreviation

CBC Convective boundary conditions

MHD Magnetohydrodynamic

\section{Introduction}

Modification and control of flow and heat transfer in an open enclosure or duct is a viable approach in various engineering applications. The convection heat transfer of an electro-conducting fluid can be controlled by using the Lorentz force by applying a magnetic field. This principle can be used for different applications in engineering such as crystal growth, metallurgical sciences, blood flow control, etc. (Lu et al. 2003).

Open enclosures or ducts can be found in numerous applications such as cooling of electronical devices, building ventilation, smoke and dust control, and microfluidics in recent years. Valencia (1999) used rectangular bars to enhance heat transfer in channels. Their data were presented for heat transfer and for different periodicity lengths in a Reynolds number range of 100-400. Lu et al. (2003) studied the enhancement of flow and heat transfer rates of air flow in a pipe. A gradient magnetic field was applied at a point of step heating in the wall. They used parameters for the strength of the magnetic field and the temperature difference between that of a pipe wall and that of inlet air. The authors found that the radial component in the velocity increased pursuant to the effect of the magnetizing force, and the thickness of velocity and thermal boundary layers decreased. Abib and Jaluria (1988) performed a numerical work on cooling of electronic components using mixed convection. They solved a conjugate problem, describing the flow and thermal fields in an air, as well as the thermal field within the walls of enclosure and the electronic components themselves. Papanicolaou and Jaluria (1994) investigated mixed convection effect from simulated electronic components at varying relative positions in an open cavity. A numerical analysis for mixed convection in a partially open rectangular cavity was performed by Gocmen and Gunes (2002). Oztop (2010) presented the influence of exit opening location on mixed convection in a channel with volumetric heat source using finite volume method.

Magnetic field can be used as a control parameter in thermo fluidics problems. It is an important heat and fluid flow for metallurgical science as given by Ozoe (2001a, 2001b). In this context, Oztop et al. (2009) studied buoyancy induced flow in magnetohydrodynamic fluid-filled enclosure with sinusoidally varying temperature boundary conditions. They found that heat transfer was decreased with increasing Hartmann number and decreasing value of amplitude of sinusoidal thermal function. Beg et al. (2009) made a theoretical study of unsteady magnetohydrodynamic viscous Hartmann-Couette laminar flow and heat transfer in a Darcian porous medium intercalated between parallel plates, under a constant pressure gradient. Viscous dissipation, Joule heating, Hall's current and non-slip current effects are included as lateral mass flux at both plates. Rahman et al. (2011) studied the effects of magnetic field on mixed convective flow in a horizontal channel with a bottom heated open enclosure with rectangular horizontal lower surface and vertical side surfaces using Galarkin weighted residual finite-element technique. Sposito and Ciofalo (2008) made a work on fully developed flow of an electrically conducting, internally heated fluid in a vertical square duct under the influence of buoyancy and magnetohydrodynamic forces. They observed the limiting values of pressure gradient and mean velocity for the flow to be unidirectional throughout the duct's section. Mahmud et al. (2003) studied analytically a combined free and forced convection flow of an electrically conducting and heat generating/absorbing fluid in a vertical channel made of two parallel plates under the action of transverse magnetic field. Oztop et al. (2012) studied MHD natural convection in an enclosure from two semi-circular heaters on the bottom wall.

The main aim of the present study is to investigate the effect of magnetic field inside a long channel having a semi-circular cavity. These types of geometries were introduced in the literature for different applications for cavities and electronic devices by Kimura et al. (1997), Oztop (2005), Chandra, and Chhabra (2011)..

\section{Analysis}

\subsection{Physical model}

The physical model under present study with the system of coordinates is depicted in Fig. 1. The problem deals with a steady two-dimensional open channel of length $L$ with semi-circular heater of diameter $0.5 L$. The flat walls are considered adiabatic and semi-circular wall is kept under the isothermal boundary conditions. It is assumed that the height of the channel $w=0.25 \mathrm{~L}$. Cold air flows through the inlet inside the channel at a uniform velocity, $u_{i}$. It is also considered that the incoming flow is at the ambient temperature $T_{i}$ and the outgoing flow is assumed to have zero diffusion flux for all dependent variables i.e. convective boundary 
conditions (CBC). The acceleration due to gravity acts in the negative y-direction. All solid boundaries are taken to be rigid no-slip walls. The magnetic-field $B_{0}$ is applied horizontally.

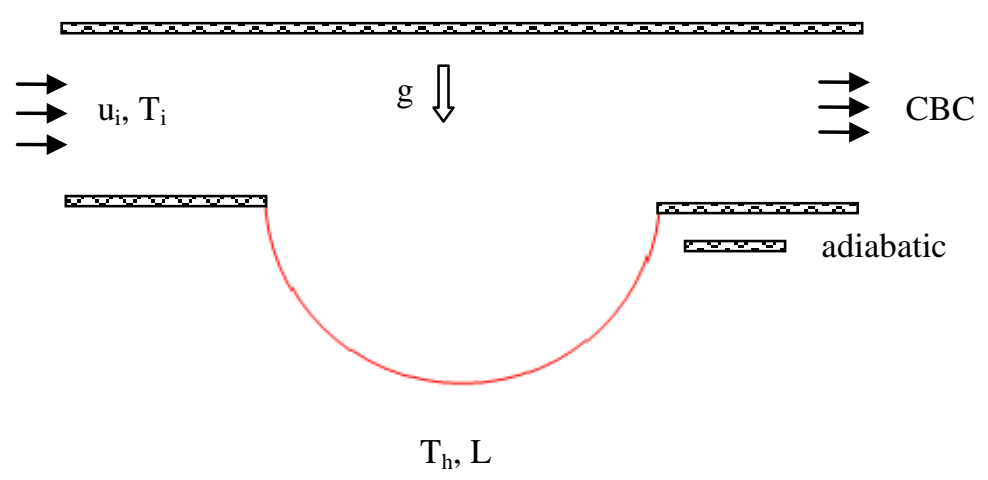

Fig.1: Physical model of the problem

\subsection{Mathematical formulation}

The governing equations for the present problem under consideration are based on the balance laws of mass, momentum and thermal energy in two dimensions. The fluid properties are also assumed constant, and the Boussinesq approximation is applied to model the buoyancy effect. The fluid is assumed Newtonian while viscous dissipation effects are negligible. The governing equations are transformed into dimensionless forms under the following non-dimensional variables (More detailed Rahman et al. (2011)):

$$
\begin{aligned}
& X=\frac{x}{L}, Y=\frac{Y}{L}, U=\frac{u}{u_{i}}, V=\frac{v}{u_{i}}, P=\frac{p}{\rho u_{i}{ }^{2}}, \theta=\frac{T-T_{i}}{T_{h}-T_{i}}, \operatorname{Re}=\frac{u_{i} L}{v}, \operatorname{Pr}=\frac{v}{\alpha}, \\
& R a=\frac{g \beta\left(T_{h}-T_{i}\right) L^{3}}{v \alpha}, H a=B_{0} L \sqrt{\sigma / \mu}, \text { and } J=\frac{\sigma B_{0}^{2} L u_{i}}{\rho c_{p}\left(T_{h}-T_{i}\right)}
\end{aligned}
$$

The dimensionless forms of the governing equations under steady state condition are expressed in the following forms (Rahman et al. (2011)):

$$
\begin{aligned}
& \frac{\partial U}{\partial X}+\frac{\partial V}{\partial Y}=0 \\
& U \frac{\partial U}{\partial X}+V \frac{\partial U}{\partial Y}=-\frac{\partial P}{\partial X}+\frac{1}{R e}\left(\frac{\partial^{2} U}{\partial X^{2}}+\frac{\partial^{2} U}{\partial Y^{2}}\right) \\
& U \frac{\partial V}{\partial X}+V \frac{\partial V}{\partial Y}=-\frac{\partial P}{\partial Y}+\frac{1}{R e}\left(\frac{\partial^{2} V}{\partial X^{2}}+\frac{\partial^{2} V}{\partial Y^{2}}\right)+\frac{R a \theta}{R e^{2} P r}-\frac{H a^{2}}{R e} V \\
& U \frac{\partial \theta}{\partial X}+V \frac{\partial \theta}{\partial Y}=\frac{1}{\operatorname{Re} \operatorname{Pr}}\left(\frac{\partial^{2} \theta}{\partial X^{2}}+\frac{\partial^{2} \theta}{\partial Y^{2}}\right)+J V^{2}
\end{aligned}
$$

The dimensionless boundary conditions corresponding to the considered problem are as follows:

at inlet: $U=1, V=0, \theta=0$

at outlet: convective boundary condition $P=0$

at all solid boundaries other than semi-circle: $U=0, V=0, \frac{\partial \theta}{\partial N}=0$

on semi-circle: $U=V=0, \theta=1$

where $N$ is the non-dimensional distances either $X$ or $Y$ direction acting normal to the surface.

The average Nusselt number evaluated along the $\mathrm{x}$-axis and $\mathrm{y}$-axis of the heated surface of the cavity based on the dimensionless quantities may be expressed respectively as

$$
N u_{x}=-\frac{1}{L_{s}} \int_{0}^{L_{s}} \frac{\partial \theta}{\partial X} d Y \text { and } N u_{y}=-\frac{1}{L_{s}} \int_{0}^{L_{s}} \frac{\partial \theta}{\partial Y} d X
$$

Drag force on the semi-circle is, $D=-\int_{0}^{L_{s}} \frac{\partial U}{\partial Y} d X$ and the average temperature of the fluid is defined as $\theta_{a v}=\int \theta d \bar{V} / \bar{V}$, where $\bar{V}$ is the cavity volume and $L_{s}$ length of the semi-circle. The non-dimensional stream function is defined as 
$U=\frac{\partial \psi}{\partial Y}, V=-\frac{\partial \psi}{\partial X}$

\section{Solution Procedure}

\subsection{Finite element technique}

The momentum and energy equations have been solved employing Galerkin finite element method. To solve the coupled governing Eqs. (2) - (4), the Penalty finite element method (Basak et al. (2010)) is used where the pressure $P$ is eliminated by a penalty constraint $\gamma$ and the incompressibility criteria given by Eq. (1) consequences in

$$
P=-\gamma\left(\frac{\partial U}{\partial X}+\frac{\partial V}{\partial Y}\right)
$$

The continuity equation is automatically fulfilled for large values of $\gamma$. Using the Eq. (5), the momentum equations $(2-3)$ is written as:

$$
\begin{aligned}
& U \frac{\partial U}{\partial X}+V \frac{\partial U}{\partial Y}=\gamma \frac{\partial}{\partial X}\left(\frac{\partial U}{\partial X}+\frac{\partial V}{\partial Y}\right)+\frac{1}{R e}\left(\frac{\partial^{2} U}{\partial X^{2}}+\frac{\partial^{2} U}{\partial Y^{2}}\right) \\
& U \frac{\partial V}{\partial X}+V \frac{\partial V}{\partial Y}=\gamma \frac{\partial}{\partial Y}\left(\frac{\partial U}{\partial X}+\frac{\partial V}{\partial Y}\right)+\frac{1}{R e}\left(\frac{\partial^{2} V}{\partial X^{2}}+\frac{\partial^{2} V}{\partial Y^{2}}\right)+\frac{R a}{R e^{2} P r} \theta-\frac{H a^{2}}{R e} V
\end{aligned}
$$

Expanding the velocity components $(U, V)$ and temperature $(\theta)$ using basis set $\left\{\Phi_{k}\right\}_{k=1}^{N}$ as

$$
U \approx \sum_{k=1}^{N} U_{k} \Phi_{k}(X, Y), V \approx \sum_{k=1}^{N} V_{k} \Phi_{k}(X, Y) \text { and } \theta \approx \sum_{k=1}^{N} \theta_{k} \Phi_{k}(X, Y)
$$

Then the Galerkin finite element method yields the following nonlinear residual equations for the Eqs. (4), (6) and (7) respectively at nodes of internal domain $\mathrm{A}$ :

$$
\begin{aligned}
& R_{i}^{(1)}=\sum_{k=1}^{N} \theta_{k} \int\left[\left(\sum_{A}^{N} U_{k} \Phi_{k}\right) \frac{\partial \Phi_{k}}{\partial X}+\left(\sum_{k=1}^{N} V_{k} \Phi_{k}\right) \frac{\partial \Phi_{k}}{\partial Y}\right] \Phi_{i} d X d Y-\frac{1}{\operatorname{RePr}} \sum_{k=1}^{N} \theta_{k} \int_{A}\left[\frac{\partial \Phi_{i}}{\partial X} \frac{\partial \Phi_{k}}{\partial X}+\frac{\partial \Phi_{i}}{\partial Y} \frac{\partial \Phi_{k}}{\partial Y}\right] d X d Y \\
& +J\left[\int\left(\sum_{A}^{N} V_{k} \Phi_{k}\right) \Phi_{i} d X d Y\right]\left[\iint_{A}\left(\sum_{k=1}^{N} V_{k} \Phi_{k}\right) \Phi_{i} d X d Y\right] \\
& R_{i}^{(2)}=\sum_{k=1}^{N} U_{k} \int\left[\left(\sum_{A}^{N} U_{k} \Phi_{k}\right) \frac{\partial \Phi_{k}}{\partial X}+\left(\sum_{k=1}^{N} V_{k} \Phi_{k}\right) \frac{\partial \Phi_{k}}{\partial Y}\right] \Phi_{i} d X d Y \\
& -\gamma\left[\sum_{k=1}^{N} U_{k} \int_{A} \frac{\partial \Phi_{i}}{\partial X} \frac{\partial \Phi_{k}}{\partial X} d X d Y+\sum_{k=1}^{N} V_{k} \int \frac{\partial \Phi_{i}}{\partial X} \frac{\partial \Phi_{k}}{\partial Y} d X d Y\right]-\frac{1}{R e} \sum_{k=1}^{N} U_{k} \int\left[\frac{\partial \Phi_{i}}{\partial X} \frac{\partial \Phi_{k}}{\partial X}+\frac{\partial \Phi_{i}}{\partial Y} \frac{\partial \Phi_{k}}{\partial Y}\right] d X d Y \\
& R_{i}^{(3)}=\sum_{k=1}^{N} V_{k} \int\left[\left(\sum_{A}^{N} U_{k} \Phi_{k}\right) \frac{\partial \Phi_{k}}{\partial X}+\left(\sum_{k=1}^{N} V_{k} \Phi_{k}\right) \frac{\partial \Phi_{k}}{\partial Y}\right] \Phi_{i} d X d Y-\gamma\left[\sum_{k=1}^{N} U_{k} \int_{A} \frac{\partial \Phi_{i}}{\partial Y} \frac{\partial \Phi_{k}}{\partial X} d X d Y+\sum_{k=1}^{N} V_{k} \int \frac{\partial \Phi_{i}}{\partial Y} \frac{\partial \Phi_{k}}{\partial Y} d X d Y\right] \\
& -\frac{1}{R e} \sum_{k=1}^{N} V_{k} \int\left[\frac{\partial \Phi_{i}}{\partial X} \frac{\partial \Phi_{k}}{\partial X}+\frac{\partial \Phi_{i}}{\partial Y} \frac{\partial \Phi_{k}}{\partial Y}\right] d X d Y-\frac{R a}{R e^{2} P r} \int_{A}\left(\sum_{k=1}^{N} \theta_{k} \Phi_{k}\right) \Phi_{i} d X d Y+\frac{H a^{2}}{R e} \int_{A}\left(\sum_{k=1}^{N} V_{k} \Phi_{k}\right) \Phi_{i} d X d Y
\end{aligned}
$$

Three points Gaussian quadrature is used to evaluate the integrals in the residual equations. The non-linear residual equations $(9-11)$ are solved using Newton-Raphson method to determine the coefficients of the expansions in Eq. (8).

\subsection{Grid refinement check and code validation}

A trial calculation is made using various non-uniform grids of triangular elements: 2322, 2780, 4068, 4926 and 6230 in order to obtain grid independent solutions as shown in Fig. 2. These experiments reveal the magnitude of average Nusselt numbers $N u_{x}$ (along x-axis) and $N u_{y}$ (along y-axis) for 4068 elements illustrates a very slight difference with the results obtained for the other elements. Finally, 4068 elements are selected for the balance between the calculation accuracy and the speed.

The present numerical code is validated against the numerical results of Manca et al. (2003) for mixed convection problem in a channel with an open cavity. The computed average Nusselt number and maximum fluid temperature for the test case are tabulated in Table 1. The agreement between the present computation and those of Manca et al. (2003) are seen to be very well with a maximum difference within $0.5 \%$. These validations make a good confidence in the present numerical code. 
(a)

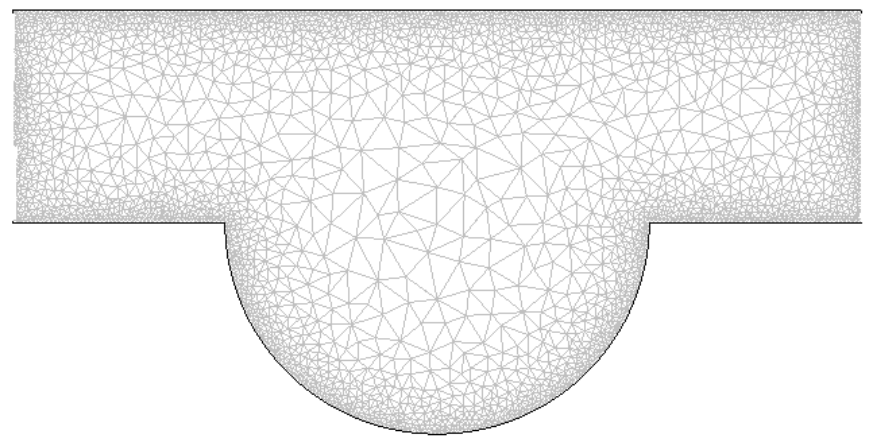

(b)

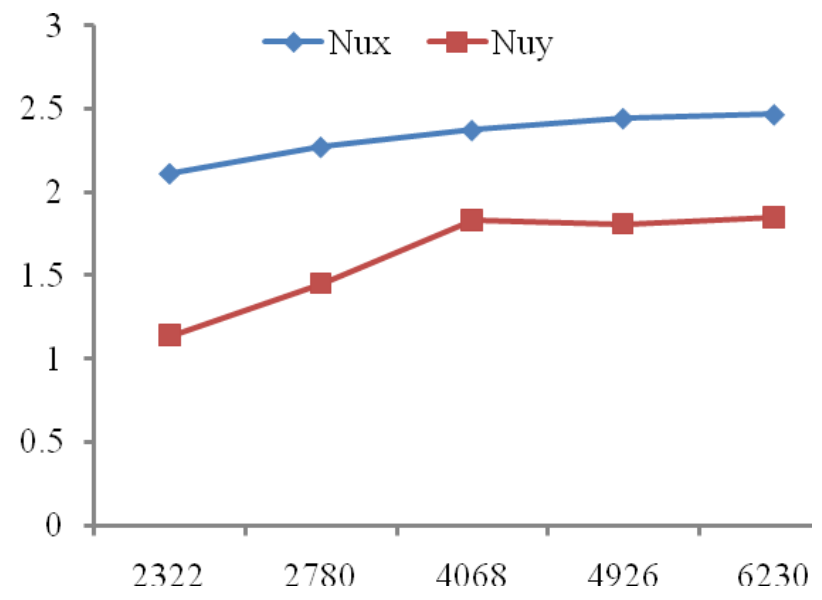

Fig. 2: (a) Grid distribution and b) Grid independency study for different grid elements while $R e=100, R a=10^{5}, \mathrm{Ha}=10$ and $\mathrm{J}=1.0$.

Table 1: Comparison of results for validation at $\mathrm{Pr}=0.71$,

$$
\mathrm{Re}=100, \mathrm{Ri}=0.1, \mathrm{w} / \mathrm{H}=0.5, \mathrm{~L} / \mathrm{H}=2
$$

\begin{tabular}{ccc}
\hline Opposing flow & Present & Manca et al. (2003) \\
\hline $\mathrm{Nu}$ & 1.7657 & 1.7748 \\
$\theta_{\max }$ & 0.629 & 0.627 \\
\hline
\end{tabular}

\section{Results and Discussion}

The present numerical study is carried out to investigate heat and fluid flow in an open channel having a semicircular heater on the bottom surface under magnetic field. The governing physical parameters in the considered problem are the Rayleigh numbers, and Hartmann numbers. In this investigation, our attention is taken into account to investigate the effects of controlling parameters namely Hartmann number ( $\mathrm{Ha}$ ), and Rayleigh numbers $(R a)$. Here, the effect of Hartmann number is investigated in the range of $10-100$ for different values of $R a\left(=10^{3}, 10^{4}\right.$, and $\left.10^{5}\right)$ while the Reynolds number, Prandtl numbers, and Joule parameters are keeping fixing as 100, 0.7, and 1.0 for whole study, respectively. Moreover, the results of this study are presented in terms of streamlines and isotherms. Furthermore, the heat-transfer effectiveness of the enclosure is displayed in terms of average Nusselt number $N u_{\mathrm{av}}$, the dimensionless average bulk temperature $\theta_{a v}$ and the drag force $D$.

\subsection{Basic features of isotherms and streamlines}

The combined forced and buoyancy driven flow and temperature fields inside the open channel, with a semicircular heater on the bottom surface for different Hartmann numbers are illustrated by means of projection of flow lines and isotherms in Figs. 3-5 for different Rayleigh numbers $R a$ of $10^{3}, 10^{4}$, and $10^{5}$, respectively. It is 
observed that conduction mode of heat transfer becomes dominant inside the cavity for low Rayleigh numbers namely $\mathrm{Ra}=10^{3}$ and $10^{4}$, (Fig 3 and 4). It is also found that the increasing of magnetic force, for instance $\mathrm{Ha}$, decreases the flow strength. This can easily be seen for higher values of high $R a$. Effectiveness of conduction becomes more effective for huge $\mathrm{Ha}$ due to suppressing of fluid by magnetic field. Both streamline and isotherms fit with the shape of semi-circular cavity. One may notice that an egg-shaped circulation cell is formed inside the semi-circular cavity in Fig. 5 at $\mathrm{Ha}=10$ due to domination of natural convection to forced convection. It is found that isotherms are deviated from its original path at this value of $R a$. When $H a$ increases to $H a=20$, the cell moves toward to left side and its length becomes smaller due to decreasing of flow strength. It disappeared dramatically for the higher values of $\mathrm{Ha}=50$, and 100 due to decreasing of domination of convection and streamlines plot with semi-circular boundary.

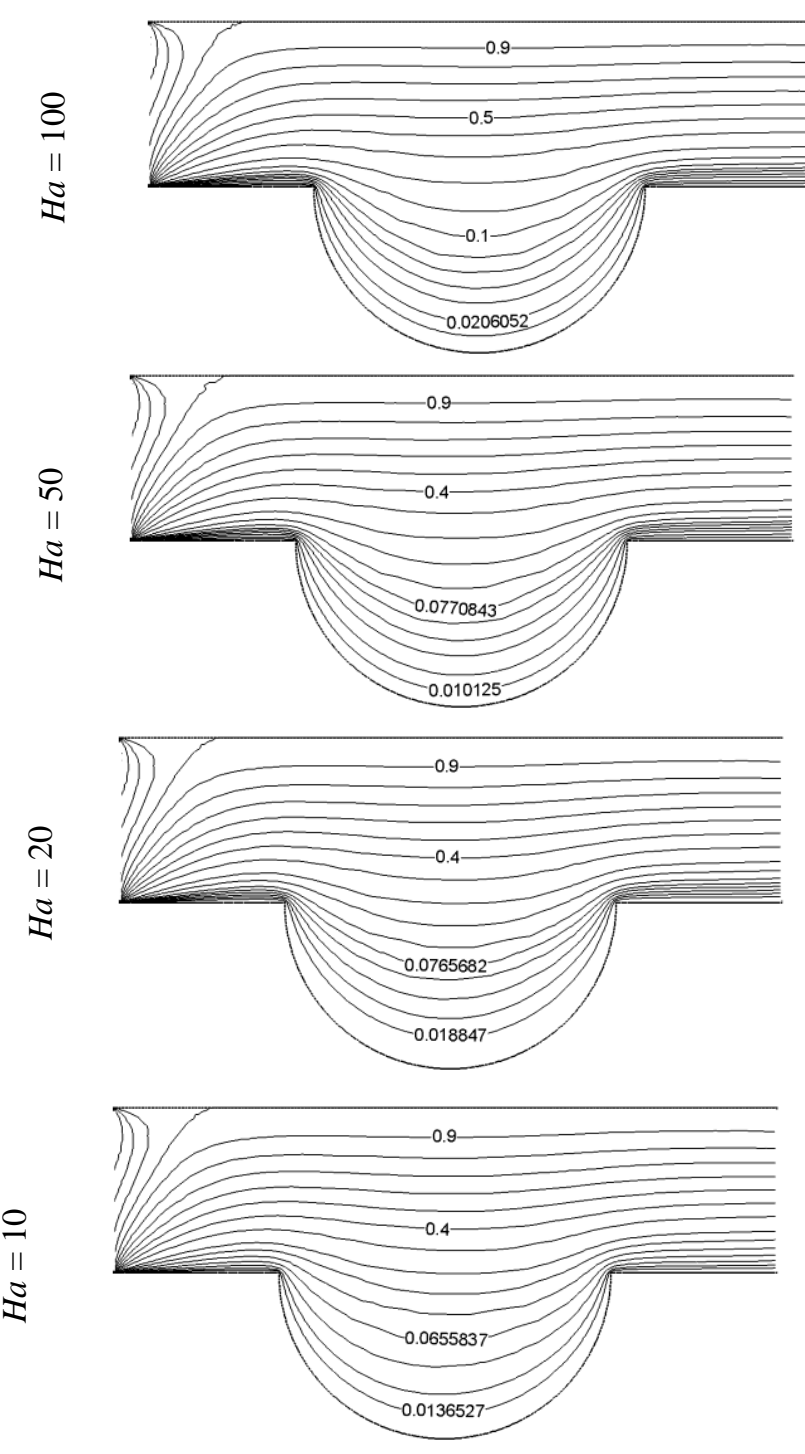

(a)
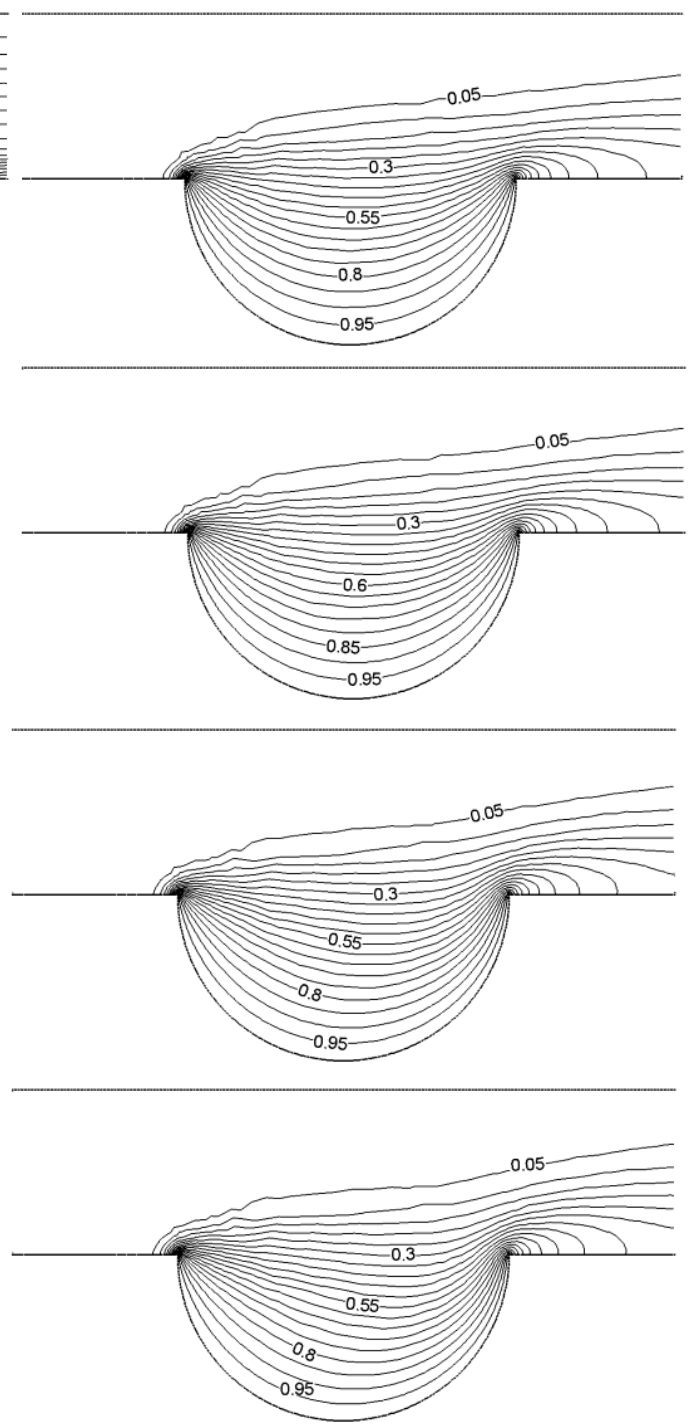

(b)

Fig. 3: (a) Streamlines and (b) Isotherms contours with $J=1.0$ and $R a=10^{3}$ at selected values of Hartmann number $\mathrm{Ha}$.

\subsection{Heat transfer effects}

The heat transfer effects are analyzed with the average Nusselt number. The effects of Hartmann number on average Nusselt number $N u_{a v}$ at the heated surface in the cavity are illustrated in Fig.6, while $\operatorname{Re}=100$ and $J=1$ 
are kept fixing. As seen from figure, $N u_{a v}$ increases with increasing of $R a$, as expected. It can clearly be seen that its value decreases with increasing of $\mathrm{Ha}$. The reason of this is retarding effect of magnetic field on convection. In addition, the slope of the curve of average Nusselt number becomes slighter for the highest value of $\mathrm{Ha}$. This is because the domination of conduction mode of heat transfers even at higher Ra.
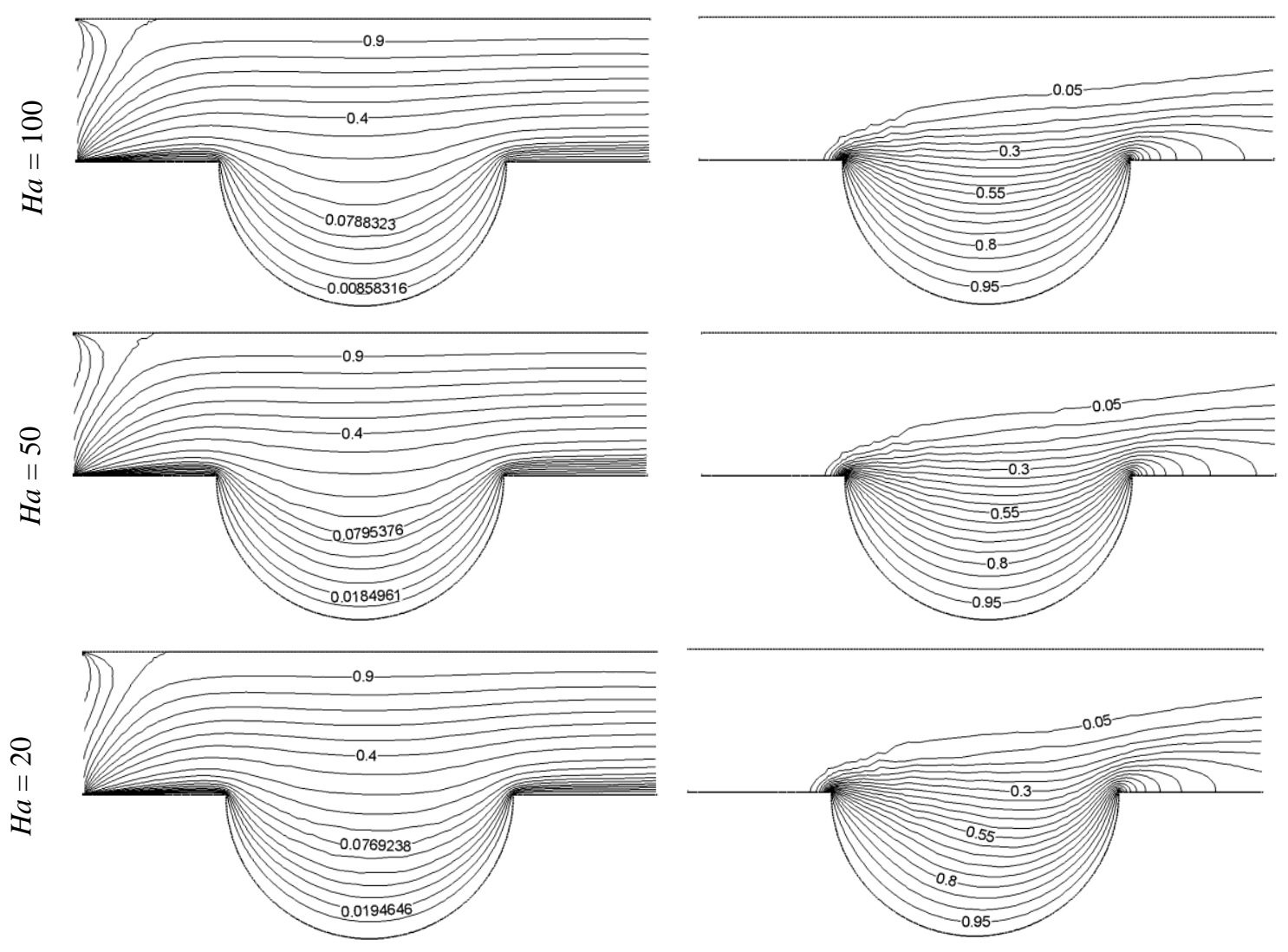

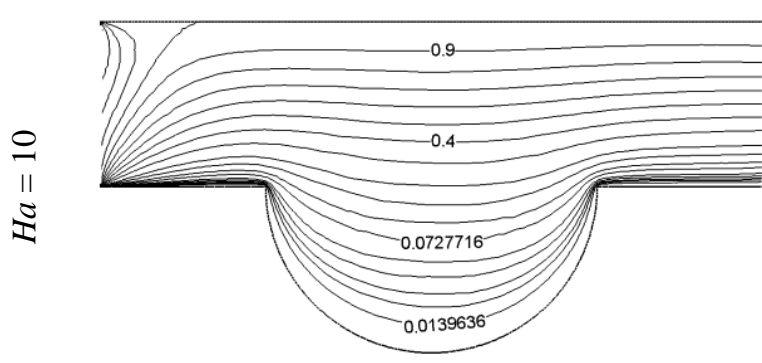

(a)

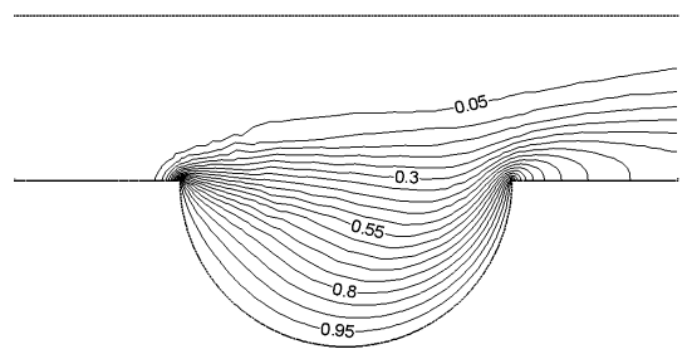

(b)

Fig. 4: (a) Streamlines and (b) Isotherms contours with $J=1.0$ and $R a=10^{4}$ at selected values of Hartmann number $\mathrm{Ha}$.

On the other hand, the effects of Hartmann number on the average bulk temperature $\theta_{a v}$ in the cavity are displayed in Fig. 7, while $R e=100$ and $\mathrm{J}=1$ keeping fixed. It is noticed that the temperature values are increased exponentially for the lower values of $H a$ (= 10 and 20). However, it is an interesting result that temperature is slightly decreased even for increasing value of $R a$ for higher values of $H a$ (= 50 and 100). For further Hartmann number, temperature will be constant with independent of Rayleigh number.

Variation of drag force with Rayleigh number is shown in Fig. 8 for different Hartmann numbers. Drag force increases quickly with increasing of Rayleigh number linearly for $H a=10$ and 20, and it becomes steady almost stationary fluid for higher values of $\mathrm{Ha}(=50$ and 100). 

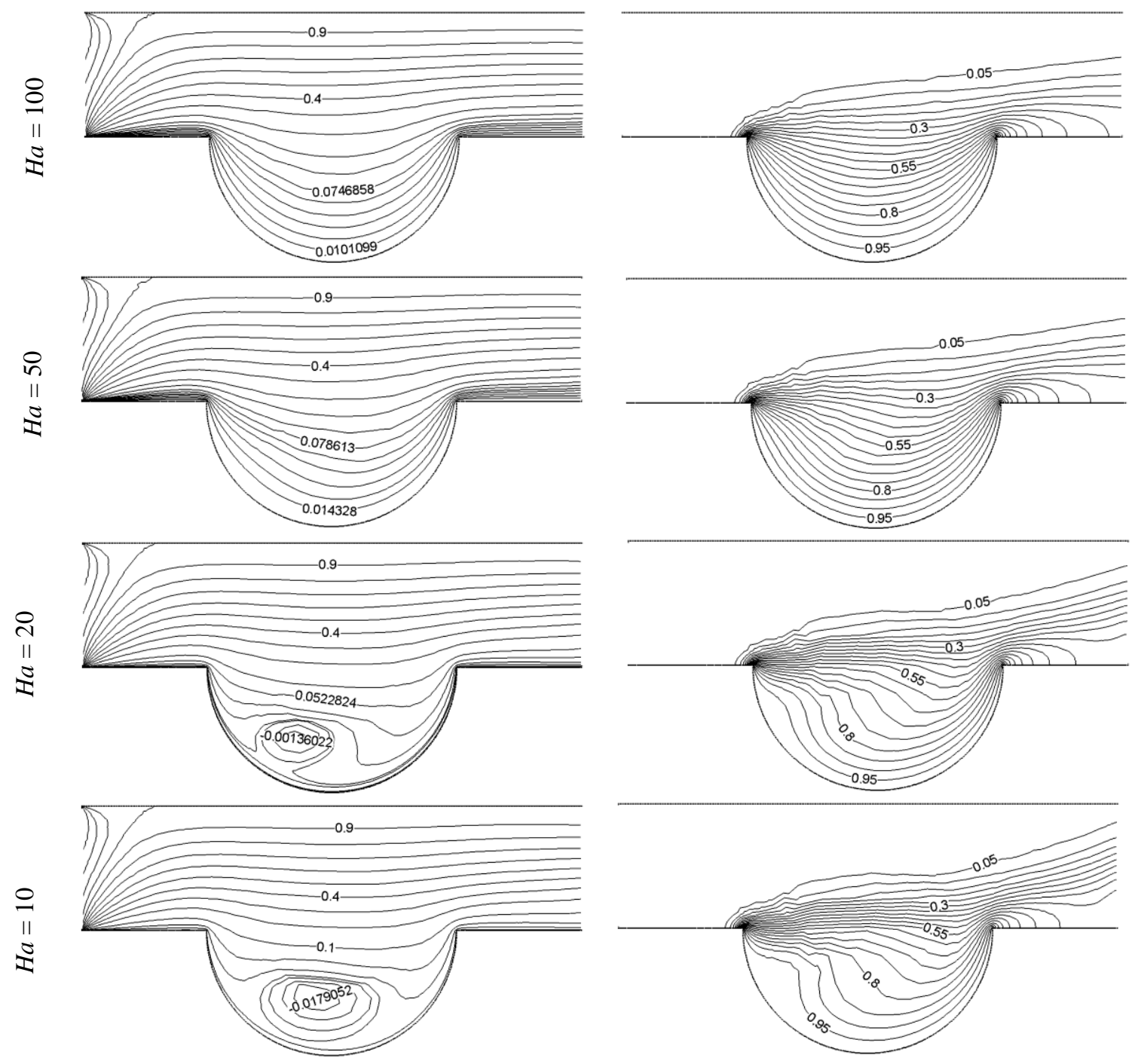

(a)

(b)

Fig. 5: (a) Streamlines and (b) Isotherms contours with $J=1.0$ and $R a=10^{5}$ at selected values of Hartmann number $\mathrm{Ha}$.

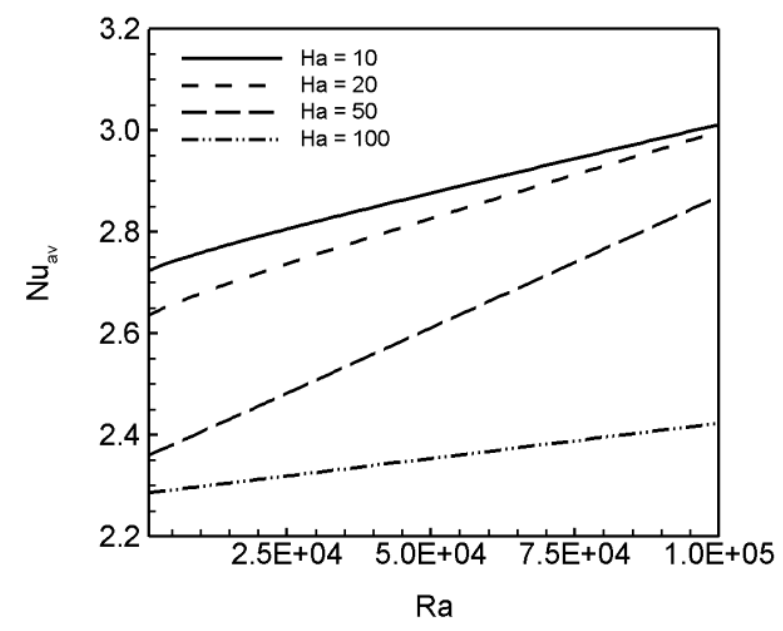

Fig. 6: Average Nusselt number versus Rayleigh number $R a$ with $J=1.0$ for different Hartmann number $H a$.

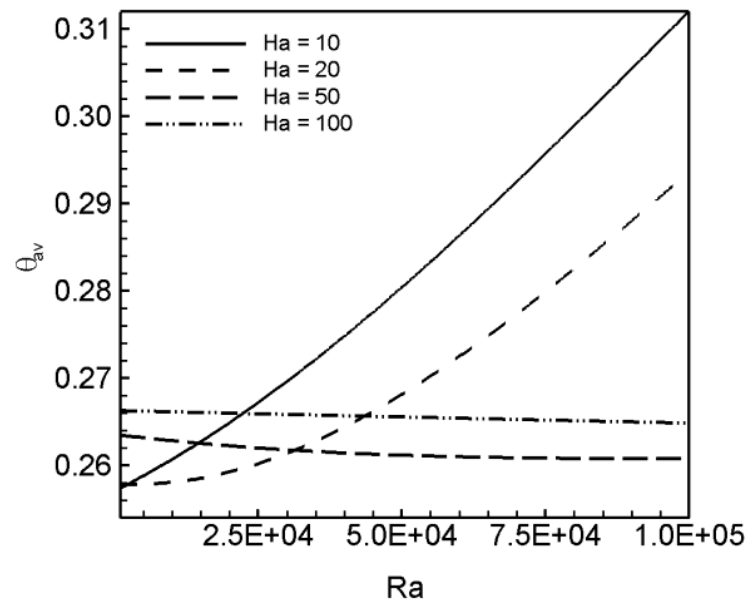

Fig. 7: Average fluid temperature versus Rayleigh number $R a$ with $J=1.0$ for different Hartmann number $\mathrm{Ha}$. 


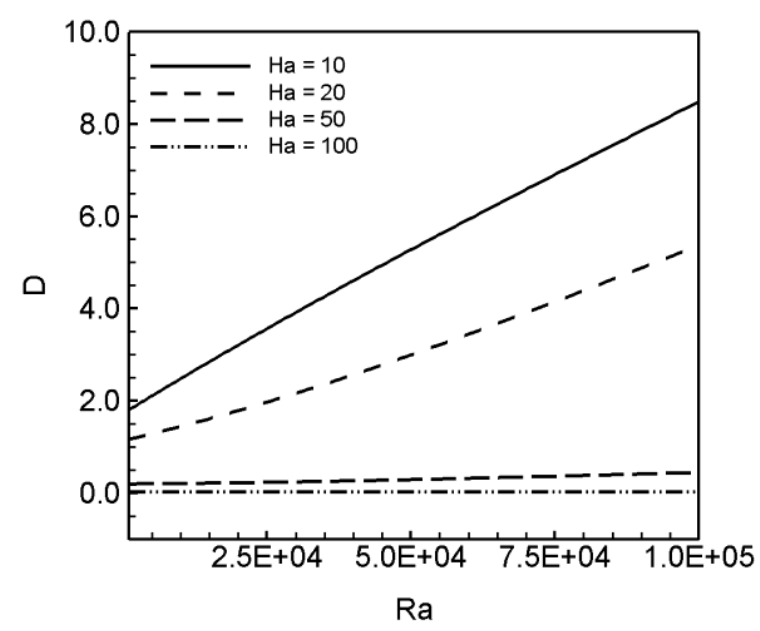

Fig. 8: Drag force versus Rayleigh number $R a$ with $J=1.0$ for different Hartmann number $H a$.

\section{Conclusion}

A numerical investigation of mixed convection heat transfer in an open channel with a semi-circular cavity has been presented. Two dimensional conservation equations of mass, momentum and energy with Boussinesq approximation have been solved using the finite-element method. The governing parameters are: $10^{3} \leq R a \leq 10^{5}$, and $10 \leq H a \leq 100$. In view of the obtained results, the following findings have been summarized:

- Streamlines and isotherms are distributed almost parallel to wall of semi-circular fashioned cavity at low $R a$ and high $H a$.

- Flow strength and heat transfer decline with increasing of magnetic force. But variation of $\mathrm{Ha}$ becomes insignificant for low $R a$.

- Drag force gives a linear increasing with $\mathrm{Ra}$ for low $\mathrm{Ha}$. Higher values of $\mathrm{Ha}$ become insignificant on Drag force.

\section{References}

Abib, A.H., and Jaluria, Y., (1988): Numerical simulation of the buoyancy-induced flow in a partially open enclosure, Numerical Heat Transfer, Part A, vol. 14, pp. 235-254.

Basak, T., Roy, S., Singh, S.K., Pop, I., (2010): Analysis of mixed convection in a lid-driven porous square cavity with linearly heated side wall(s), International Journal of Heat and Mass Transfer, vol. 53, pp. 1819-1840. doi.10.1016/j.ijheatmasstransfer.2010.01.007

Bég, O.A., Zueco, J., Takhar, H.S., (2009): Unsteady magnetohydrodynamic Hartmann-Couette flow and heat transfer in a Darcian channel with Hall current, ionslip, viscous and Joule heating effects: Network numerical solutions, Communication in Nonlinear Science and Numerical Simulation, vol. 14, pp.1082-1097. doi.10.1016/j.cnsns.2008.03.015

Chandra, A., and Chhabra, R.P., (2011): Flow over and forced convection heat transfer in Newtonian fluids from a semi-circular cylinder, International Journal of Heat and Mass Transfer, vol. 54, pp.225-241. doi.10.1016/j.ijheatmasstransfer.2010.09.048

Gocmen, K., and Gunes, H., (2002): Mixed convection in a partially open rectangular cavity, $6^{\text {th }}$ Biennial Conf. Eng. Systems Design and Analysis, Proc. ESDA 2002, ESDA/ATF-003. 
Kimura, T., Takeuchi, M., Nagai, N., Hirano, M., (1997): Experimental study of natural convection heat transfer in a semicircular enclosure, Heat Transfer-Japanese Research, vol. 26, pp. 131-142. doi.10.1002/(SICI)15206556(1997)26:2<131::AID-HTJ7>3.0.CO;2-X

Lu, S.S., Lee, C.H., Tagawa, T., Ozoe, H., and Hua, B., (2003): Enhancement of heat transfer and air flow rates in a pipe with application of a magnetic field, Journal of Enhanced Heat Transfer, vol. 10, pp. 45-60. doi.10.1615/JEnhHeatTransf.v10.i1.50

Mahmud, S., Tasnim, S.H., Mamun, M.A.H., (2003): Thermodynamic analysis of mixed convection in a channel with transverse hydromagnetic effect, International Journal of Thermal Science, vol.42, pp. 731-740. doi.10.1016/S1290-0729(03)00040-1

Manca, O., Nardini, S., Khanafer, K., Vafai, K., (2003): Effect of heated wall position on mixed convection in a channel with an open cavity, Journal of Numerical Heat Transfer vol. 43 (3), pp. 259-282. doi.10.1080/10407780307310

Ozoe, H., (2001a): Natural and mixed convection of liquid metals in a magnetic field, CHT'01 Adv. Comput. Heat Transfer, vol. II, pp.139-153.

Ozoe, H., (2001b): Melt stirring effect of a weak magnetic field on crystal growth, Progress in Crystal Growth and Characterization of Materials., vol. 38, pp. 239-259. doi.10.1016/S0960-8974(99)00014-5

Oztop, H.F., (2005): Effective parameters on second law analysis for semicircular ducts in laminar flow and constant wall heat flux, International Communication in Heat and Mass Transfer, vol. 32, pp. 266-274. doi.10.1016/j.icheatmasstransfer.2004.05.018

Oztop, H.F., (2010): Influence of exit opening location on mixed convection in a channel with volumetric heat sources, International Communication in Heat and Mass Transfer, vol. 37, pp. 410-415. doi.10.1016/j.icheatmasstransfer.2010.01.006

Oztop, H.F., Oztop, M., Varol, Y., (2009): Numerical simulation of magnetohydrodynamic buoyancy-induced flow in a non-isothermally heated square enclosure, Communication in Nonlinear Science and Numerical Simulation, vol. 14, pp.770-778. doi.10.1016/j.cnsns.2007.11.005

Oztop, HF., Rahman, M.M., Ahsan, A., Hasanuzzaman, M., Saidur, R., Khaled Al-Salem, Rahim N.A. (2012): MHD natural convection in an enclosure from two semi-circular heaters on the bottom wall, International Journal of Heat and Mass Transfer, vol. 55, pp. 1844-1854. doi.10.1016/j.ijheatmasstransfer.2011.11.037

Papanicolaou, E., and Jaluria, Y., (1994): Mixed convection from simulated electronic components at varying relative positions in a cavity, Journal of Heat Transfer, vol. 116, pp. 960-970. doi.10.1115/1.2911472

Rahman, M.M., Parvin, S., Saidur, R., Rahim, N.A., (2011): Magnetohydrodynamic mixed convection in a horizontal channel with an open cavity, International Communication in Heat and Mass Transfer, vol. 38, no. 2, pp. 184-193. doi.10.1016/j.icheatmasstransfer.2010.12.005

Sposito, G., Ciofalo, M., (2008): Fully developed mixed magnetohydrodynamic convection in a vertical square duct, Numerical Heat Transfer A vol. 53, pp.907-924. doi.10.1080/00397910601149934

Valencia, A., (1999): Heat transfer enhancement due to self sustained oscillating transverse vortices in channels with periodically mounted rectangular bars, International Journal of Heat and Mass Transfer, vol. 42, pp.20532062. doi.10.1016/S0017-9310(98)00295-6 